\title{
Magdalena MaLinowsKa
}

Université de Silésie à Katowice

(iD) 0000-0002-0007-9017

\section{Représentations stéréotypées de la femme célibataire chez Leïla Marouane et Kaouther Adimi}

\author{
Stereotypical representations of a single woman in novels \\ by Kaouther Adimi and Leïla Marouane
}

\begin{abstract}
In this paper, the author analyzes the stereotypical representations of single woman from two French-language Algerian novels: Les Pierres dans ma poche by Kaouther Adimi and La fille de la Casbah by Leilla Marouane. By using different literary techniques, the chosen writers formulate a severe criticism of contemporary Algerian society which, despite the hard-won liberties of the second sex, maintains the status quo by enclosing women in stereotypical social roles and by stigmatizing those who do not do not conform to societal standards.
\end{abstract}

Key Words: stereotype, spinster, francophone literature, Algeria, feminism

«La vieille fille est la femme qui n'a plus aucune chance de rencontrer l'homme qui la façonne... elle est d'autant plus méchante qu'elle avance dans son état de vieille fille» (Weininger, 1920; cité d'après Perrig-Chiello, 2001 : 78). L'image d'une femme célibataire aux formes généreuses et à la beauté fanée, amère dans sa solitude, continue à persister dans l'imaginaire collectif, de même que les préjugés qui l'accompagnent. Cette image stéréotypée prend sa source dans le rôle social traditionnellement assigné à la femme, celui d'épouse et de mère, reléguée à l'espace du foyer, chargée des tâches domestiques. Certes, les femmes jouissent à présent des libertés durement acquises, telles que le droit à l'instruction et à l'emploi, ce qui leur a permis de sortir des quatre murs de la maison. Néanmoins, malgré ces avancées, le mariage et la famille constituent l'idéal de la vie adulte dans les sociétés contemporaines, "le modèle de toute vie» (HÉritier, 1996 : 239). Cela est surtout visible dans les sociétés traditionnelles, notamment dans l'aire culturelle arabo-musulmane, dans laquelle 
«le mariage reste primordial» ( $« 97 \%$ d'homme et de femmes en contractent au moins un au cours de leur vie») (Rude-Antoine, 1990: 25). Cela s'ensuit de la religion musulmane dont le livre sacré, le Coran, réprime le célibat et incite la communauté à marier ses membres: «Mariez les célibataires qui sont parmi vous, ainsi que ceux de vos serviteurs des deux sexes qui pratiquent la vertu » (Sourate 24, verset 32).

$\mathrm{Au} \mathrm{XXI} \mathrm{I}^{\mathrm{e}}$ siècle, l'impératif de s'unir et la pression sociale qui pèse sur les personnes célibataires commencent à être remis en cause en tant que contraintes au libre épanouissement de chaque personne. Ces phénomènes sont notamment critiqués par les romancières algériennes contemporaines pour lesquelles la lutte contre les traditions rétrogrades et pour l'indépendance de la femme reste primordiale. Leur combat passe entre autres par la dénonciation du rôle assigné traditionnellement à la femme et cela par la ridiculisation et la contestation du stéréotype de la célibataire, la «vieille fille», la baïrra.

Parmi ces Algériennes de plume, nous avons choisi pour notre étude Kaouther Adimi, une jeune romancière déjà reconnue par la critique, et Leïla Marouane, d'une trentaine d'années son aînée, appelée «la plus rebelle des écrivaines arabes 》 (EL Nossery, 2012: 138). Nous voudrions soumettre à l'analyse deux romans des auteures ci-mentionnées: La fille de la Casbah de Marouane, publié en 1996 aux éditions Julliard, et Les pierres dans ma poche d'Adimi, publié vingt ans plus tard aux éditions du Seuil. Les œuvres choisies diffèrent à bien des égards, mais elles ont en commun la place centrale assignée à la question du mariage. Bien que leur action se place dans des époques et endroits bien distincts - Alger des années quatre-vingts du $\mathrm{XX}^{\mathrm{e}}$ siècle et Paris de la seconde décennie du $\mathrm{XXI}^{\mathrm{e}}$ siècle -, l'importance du mariage, de la maternité et la fonction sociale attribuée à la femme restent quasiment inchangés. Les protagonistes de ces romans, Hadda et une héroïne anonyme, se ressemblent en ce qu'elles se trouvent coincées entre les attentes de la société - trouver un mari et fonder une famille -, et leurs aspirations personnelles: pour l'une c'est le passage de la misère à la vie luxueuse, pour l'autre la carrière et le «vrai amour».

Ce qui nous intéresse essentiellement dans notre étude ce sont les représentations stéréotypées de la femme célibataire. La notion de stéréotype, que nous utilisons telle qu'elle est définie dans les sciences sociales, désigne une «représentation collective [...] constituée par l'image simplifiée d'individus, d'institutions ou de groupes» (WILLEMs, 1970: 277) ou encore un "schème récurrent et figé en prise sur les modèles culturels et les croyances d'une société donnée» (Amossy, 1989: 36). Le stéréotype se caractérise par une forte fréquence, le figement, l'absence d'origine repérable, la prégnance dans la mémoire collective, le caractère abstrait et synthétique (DufaYs, 1994, en ligne). Bien qu'il permette de catégoriser et d'ordonner le monde qui nous entoure, il renvoie également aux préjugés qui peuvent engendrer l'ignorance, voire le dédain, et attiser des antagonismes entre individus ou entre groupes sociaux. Comme le remarquent Ruth 
Amossy et Anne Herschberg Pierrot, il est naturel d'attribuer aux gens certains traits de caractère, des comportements, des habitudes selon leur appartenance à un groupe ethnique, religieux, professionnel, etc. Elles préviennent cependant qu'en stéréotypant les membres d'un groupe, on confond la conséquence avec la cause : "on rapporte à une essence immuable des traits qui dérivent en fait de leur statut social ou des rôles sociaux qui leur sont conférés » (Amossy, HersCHBERg Pierrot, 1997 : 38). Et pour illustrer leur propos, les chercheuses donnent l'exemple de la femme :

[...] les comportements de la femme reflètent des rôles sociaux : ce qu'on attend d'elle détermine ses façons de faire et d'être. Elle apparaît ainsi comme soucieuse de bien-être de son entourage et dévouée [...]. Il ne s'agit pas là de traits innés définissant la féminité comme telle, mais des effets de la distribution sociale des rôles entre les sexes.

Comme nous l'avons déjà souligné, même si, de nos jours, la femme possède un vaste éventail de fonctions sociales à exercer, celle d'épouse et de mère remporte sur toutes les autres. Ainsi peut-on établir trois catégories de femmes selon leur statut matrimonial: les femmes mariées qui ont une existence sociale reconnue, les jeunes filles en recherche de ce statut durable et les «vieilles filles », celles qui se trouvent à la périphérie de la société. Dans la présente étude, nous allons analyser des représentations de cette dernière catégorie issues de notre corpus.

Le roman d'Adimi est construit autour d'un événement familial à venir, à savoir des fiançailles de la sœur cadette de l'héroïne anonyme, événement qui orientera dès lors les actions entreprises par la protagoniste. La nouvelle lui est annoncée par la mère lors d'une conversation téléphonique :

- Qu'est-ce qu'il y a maman?

- J'ai une grande nouvelle à t'annoncer, il fallait que je t'appelle. Je suis tellement contente: ta petite sœur va se marier!

$-\ldots$

- Tu as entendu? Il ne reste que toi à marier!

Adimi, 2016: 16

Cette remarque peu délicate de la mère, «Il ne reste que toi...», qui rappelle à l'héroïne son statut de brebis galeuse de la famille, va revenir au cours du récit comme une incantation. Ainsi, subissant à la pression, la protagoniste tente l'impossible et s'efforce de trouver un partenaire en un mois, avant son retour en Algérie natale.

${ }^{1}$ Dorénavant, les citations des Pierres dans ma poche (édition mentionnée dans la bibliographie) seront indiquées par le sigle $P P$, suivi de la pagination. 
Il en est autrement dans La fille de la Casbah, bien que son intrigue se tisse aussi autour de la question du mariage. À la différence de la protagoniste adimienne, Hadda a déjà un candidat à sa main, son cousin Brahim, que sa mère aime "comme on aime l'unique prétendant de sa fille» (Marouane, 1996: 17²). Néanmoins, l'héroïne songe à une vie libre, luxueuse et moderne, et surtout à quitter (peu importe le prix) sa Casbah natale. Elle rejette ainsi la proposition de mariage de Brahim à cause de sa gentillesse, de son traditionalisme, peut-être aussi de sa pauvreté, en lui préférant Nassib, un homme certes déplaisant et laid, mais riche et occidentalisé.

Les deux héroïnes sont des trentenaires célibataires et sont considérées comme «vieilles filles » par leur entourage. Généralement, dans les pays du Maghreb, le célibat est jugé indésirable, surtout dans le cas des femmes sur qui pèse l'impératif de la maternité. Isabelle CHARPENTIER (2015:6) remarque que, même si «le célibat féminin progresse nettement depuis le milieu des années quatrevingt-dix», il n'est pas socialement accepté, surtout par la famille qui le traite comme un "grand malheur» - en conséquence, il est difficilement vécu par la célibataire. C'est le cas des deux héroïnes dans notre corpus, qui subissent toutes les deux une forte pression sociale qui est omniprésente et se manifeste de différentes manières. Elle vient de la part de la famille proche et éloignée, mais avant tout de la mère pour qui marier sa fille constitue une des obligations parentales essentielles, et quand son enfant avance dans l'âge et risque d'être considérée comme une «vieille fille», cette obligation devient urgente. Les deux génitrices de notre corpus traitent très sérieusement la menace de célibat prolongé planant sur leurs progénitures. La mère de Hadda se montre déchirée entre les sentiments de fierté et de peur de voir sa fille unique célibataire: "Mes yeux rougis par les larmes inquiétaient toujours ma mère. [...] Qui voudrait d'une fille aveugle, même si l'adoration d'Allah en était la cause? Et ma mère de se lamenter sur mes trente ans» ( $F C 12)$. La femme est désespérée, car, venant d'un milieu très traditionnel et conservatif, elle voudrait que sa fille non seulement se marie, mais qu'elle le fasse conformément aux traditions et à la religion. En plus, elle se doute que Hadda transgresse les lois ancestrales en entretenant une relation illicite et sans avenir et elle craint que sa seule fille ne s'y perde en couvrant la famille de honte. Marouane montre avec habileté comment la piété de ce personnage se conjugue avec la croyance en magie:

Selon les visions de la doyenne, quelqu'un - la mère de mon cousin Brahim, avait-on déduit - avait accroché une amulette contenant une mèche de mes cheveux et un petit morceau d'un de mes linges sur l'aile d'une hirondelle. J'étais devenue comme l'oiseau, fendant le ciel en quête de chaleur. Telle était - entre autres - l'explication de mon passage de la piété à la mauvaise vie.

${ }^{2}$ Pour les citations qui vont suivre, le sigle $F C$ désignera La fille de la Casbah et les chiffres, le numéro de la page. 
Il ne faut pas oublier que, dans la conception patriarcale de la société, la femme en tant que mère joue un rôle symbolique de premier plan, celui de «gardienne de l'honneur des hommes» (Charpentier, 2012: 206). Et en Algérie, comme dans d'autres sociétés traditionnelles, cet honneur dépend directement de la conduite morale des femmes, surtout de la virginité des jeunes filles qui « constitue la pierre angulaire de l'éducation féminine dans le milieu traditionnel maghrébin» (CHEbel, 2003: 99). Cette éducation vise l'intériorisation des modèles de comportement valorisés positivement par la communauté. La mère de Hadda qui fait partie des "Gardiennes, les vigiles de l'ordre et des traditions » ( $F C 12$ ) est consciente des conséquences de l'insoumission aux mœurs et supplie sa fille de terminer sa relation illicite: «Je t'en prie, pense aux voisins. Pense à ce que vont dire de nous les voisins. Pour la mémoire de ton père, pour la moustache de tes frères, pense à la honte que tu nous infliges. » (FC 136) Elle craint les médisances de l'entourage puisqu'elle sait bien que la réputation irréprochable de la femme est une condition indispensable pour le mariage: « un hymen intact demeure, dans de nombreux cas, le premier capital féminin convertible et rentable sur le marché matrimonial» (CHARPENTIER, 2012: 202). Elle ne veut pas que sa fille unique compromette toutes ses chances de se marier et reste à vie une vieille fille déshonorée.

Cette tutelle des femmes sur leurs filles, qui peut paraître rétrograde au $\mathrm{XXI}^{\mathrm{e}}$ siècle, est moquée par Adimi. La narratrice met à nu l'hypocrisie de sa propre mère qui d'une gardienne des traditions est devenue presque une agente matrimoniale :

On est forcé d'écouter sa mère nous parler des risques qu'encourt une jeune fille bien à fréquenter un garçon, qu'il soit de bonne ou de mauvaise famille. Elle m'a parlé de Dieu, du Diable, de mes règles, des filles qui jetaient l'opprobre sur leur entourage, de la dignité de mon père et d'autres choses encore. Dix ans plus tard, elle me tiendra le discours inverse et m'expliquera que la dignité de mon père n'était pas ce que je croyais.

Avec de l'humour qui constitue le trait dominant de son caractère, la narratrice révèle le paradoxe de l'éducation traditionnelle qui consiste d'abord à protéger les filles de tout contact avec les garçons qui pourrait jeter un doute sur leur bonne conduite et ensuite à les pousser dans les bras de n'importe quel homme quand elles ont dépassé l'âge jugé comme convenant pour le mariage.

Ce n'est pas d'ailleurs la seule fois que l'héroïne dénonce l'absurdité de certaines règles sociales, comme cette catégorisation des filles :

Il y a donc deux types de filles. Les filles bien et les autres. Pour déterminer si une fille est bien ou pas, il suffit de tendre l'oreille et d'écouter son rire. $\mathrm{Si}$ c'est un rire en hahaha, elle fait partie des autres. En revanche, si c'est un rire 
en hihihi, on peut être rassurée et considérer qu'elle fait partie du très sélectif club des filles bien.

Et elle ajoute un peu plus tard: "À retenir: une jeune fille de bonne famille boit des jus de pamplemousse et rit en faisant hihihi» ( $P P$ 63). Elle se moque ainsi des us et coutumes qui divisent et jugent les êtres sur des critères aussi superficiels que la façon de rire.

Les génitrices des deux romans ne cachent ni l'attente de voir leur fille mariée, ni leur inquiétude, voire leur découragement face à cette question. Telle est au moins l'attitude de la mère dans Les pierres dans ma poche pour qui le mariage constitue le but de la vie de la femme ( $P P$ 131). Elle oscille entre la volonté de convaincre sa fille des bienfaits du mariage en lui disant par exemple: «Tu sembles fatiguée. Tu travailles trop. Il te faut te marier.» (PP 164), et le doute sur la possibilité de marier son aînée, qu'elle ne feint d'ailleurs guère: «J'ai pensé que tu pourrais profiter de l'occasion [des fiançailles de la cadette] pour porter le caftan que ton père et moi t'avons offert. On l'avait acheté pour ton mariage mais ce n'est pas prévu pour le moment, il faut voir la réalité en face, tu n'es pas près de te marier...» ( $P P$ 99). Des taquineries de ce type, assez caractéristiques pour le personnage de la mère, sont appelées par la narratrice « des missiles»: «La seule chose qui importe est de définir le plus vite possible le type de missile. Est-ce l'un de ceux qui m'explosera immédiatement au visage ou une arme plus sophistiquée qui empoisonnera mon esprit et mon cœur avant de m'achever douloureusement?» (PP 97-98) Nous pouvons constater que les reproches de la mère ne laissent pas l'héroöne indifférente, malgré la distance physique qui les sépare. Le téléphone, à l'instar du cordon ombilical virtuel, permet à la génitrice de contrôler de loin sa fille expatriée et empêche en même temps celle-ci de jouir pleinement de son indépendance. Qui pis est, les continuels appels de l'Algérie non seulement rappellent à la protagoniste son statut de célibataire, mais aussi lui mettent de plus en plus de pression pour en terminer.

Marouane et Adimi attirent également notre attention sur un autre problème, à savoir l'indépendance relative des jeunes algériennes par rapport aux générations précédentes et les dissentiments qui en résultent. Étant donné que dans la société de jadis l'éducation, de même que le travail en dehors de la maison, étaient réservés aux hommes, la plupart des femmes n'avaient pas de possibilité de se former, elles étaient enfermées dans les demeures durant toute leur vie. Avec l'arrivée de l'indépendance en Algérie, les jeunes filles ont eu l'accès à l'instruction. Hadda fait partie de cette génération d'«enfants de la liberté» (FC 18), mais, contrairement à plusieurs filles de son milieu, elle a pu effectuer non seulement la scolarité primaire, mais aussi terminer les études supérieures. Cette tendance s'est largement répandue et, de nos jours, le taux d'alphabétisation des femmes est très élevé et les disproportions entre les filles et les garçons 
à tous les niveaux de l'enseignement sont minimes (cf. MaLinowskA, 2019). Néanmoins, comme le constate Isabelle Charpentier :

[...] l'instruction et l'occupation d'un emploi n'augmentent pas la probabilité pour une Maghrébine de se marier, bien au contraire, pas plus qu'elles ne garantissent, aujourd'hui comme hier, une homogamie sociale et intellectuelle en cas d'union, y compris pour les générations de femmes nées après la décolonisation.

Charpentier, $2015: 5$

Son observation est confirmée dans les deux romans. Quoique la mère de Hadda soit fière de l'érudition de sa fille, appelée "Hadda la Sage » (FC 12), elle craint en même temps que ce trop d'instruction soit non un atout, mais une entrave au mariage. Elle observe qu'autour d'elle les filles qui ont vite abandonné l'école se marient sans problème pendant que sa fille reste célibataire:

Je n'écoutais plus ma mère, partie, comme chaque fois qu'elle en avait l'occasion, à énumérer les qualités des filles de la maison. [...] Et ma mère ne manquait pas de rappeler qu'aucune d'elles n'avait même pas été au lycée! - Toutes se marieront, pourtant. Et avec les honneurs, soupira-t-elle, ravalant ses larmes.

FC 118-119

Quant à l'héroïne adimienne, son incapacité à trouver un partenaire est de même mis sur le compte de son instruction et d'excès d'indépendance: «Tu as fait trop d'études, ça effraie tout le monde. Arrête de parler, tu parles trop. Ne dis pas à tout le monde que tu habites toute seule, que vont croire les gens?» ( $P P$ 98), la réprimande la mère. Ainsi, les écrivaines observent qu'il est très difficile de changer les mentalités et que, dans les sociétés traditionnelles, les femmes qui sont en avance sur leur temps seront confrontées à l'incompréhension de l'entourage.

De cette manière on essaie de convaincre toutes les femmes de se conformer au même moule. La pression pour se marier et fonder une famille vient notamment de la part de l'entourage - des voisines, des collègues, forçant les jeunes célibataires à se consacrer à leur "vocation », comme dans le cas de la protagoniste de La fille de la Casbah: "aucune [des voisines] ne manqua de rappeler ce qu'il me restait à faire : trouver un mari, un homme intelligent et sage, disaient les plus jeunes, la larme à l'œil. Engendrer une progéniture solide et nombreuse, disaient les plus âgées» ( $F C$ 81). Les femmes de la Maison des jasmins dans laquelle vit l'héroïne la félicitent d'avoir terminé les études, mais en même temps traitent cette étape comme un prélude à la «vraie vie», la vie familiale. Ainsi, si l'on tolère le fait qu'une femme étudie ou travaille, cela ne peut pas se faire au détriment de ce rôle majeur d'épouse et de mère. 
La société algérienne non seulement incite ses membres à se marier, mais encore elle réprime sévèrement le célibat. Cela s'ensuit de la religion musulmane, comme le rappelle à Hadda une de ses collègues en citant la sunna ${ }^{3}$ : "Ceux qui vivent en célibataire sont de la pire espèce; ceux qui meurent en célibataire sont de la plus ignoble» ( $F C 40)$. Choisir volontairement de ne pas se marier, ne pas avoir de famille, d'enfants, est considéré dans la culture musulmane comme irrationnel, voire absurde. Le célibat, même s'il est choisi par la femme et non imposé, peut parfois générer chez elle «le sentiment d'incomplétude et l'impossible reconnaissance socio-familiale [...], quels que soient ses réussites scolaires et ses succès professionnels» (ChARPENTIER, 2015: 8). En d'autres termes, aucune autre fonction sociale, aussi importante qu'elle soit, ne peut, dans les yeux de la communauté, récompenser le manque de progéniture. En témoigne également la réaction d'un collègue de Hadda, lui aussi professeur au lycée, à son célibat: "Comme tous ceux du lycée, du gardien aux élèves, cet homme me plaignait - dans son regard la pitié et le reproche se mêlaient, s'il ne me blâmait pas, de travailler au lieu de me consacrer à l'éducation d'une famille, de ma famille, ma fonction naturelle» (FC 39). Ainsi la maternité est-elle considérée comme la seule vocation de la femme, vocation résultant directement de son anatomie en tant que celle qui peut porter et mettre au monde un enfant, et indirectement de son importance dans le processus d'élever cette progéniture. Et la narratrice ajoute :

Ce que vouait en réalité l'intendant, c'est que Mlle Bouchnaffa avait largement passé l'âge d'avoir une vie «normale». J'étais devenue une baïrra, une vieille fille, j'allais croupir sur les estrades du lycée, assistant, impuissante, au déferlement de quelques générations. Et ma matrice se ratatinerait comme une peau de chèvre au soleil.

FC 39

Hadda se sert du mot baïra qu'elle traduit comme «vieille fille». Ce vocable vient de l'arabe classique روب qui signifie «friche» et se réfère surtout au contexte agricole pour désigner une terre non cultivée, abandonnée, infertile. En arabe algérien, le mot baïrra est employé comme une injure, pour stigmatiser une femme qui n'a pas de mari et, par conséquent, son corps ne peut pas « porter de fruit», enfanter. Cette image d'infertilité est renforcée dans l'extrait cité par la comparaison de l'utérus de Hadda à «une peau de chèvre» déformée par la chaleur.

Rappelons que l'action de La fille de la Casbah se déroule dans les années quatre-vingt. On pourrait donc penser que vingt-cinq ans plus tard, déjà au $\mathrm{XXI}^{\mathrm{e}}$

${ }^{3}$ Sunna - «Dans la religion islamique, „Coutume orthodoxe du Prophète, de ses Compagnons, des quatre premiers califes 'bien dirigés' et des Suivants immédiats” (Dermenghem)» (Grand Robert électronique, 2009). 
siècle, la femme peut décider si elle veut s'unir avec quelqu'un pour la vie ou non. L'héroïne des Pierres dans ma poche constate le contraire et dénonce elle aussi la pression qu'elle subit de la part de son entourage. Elle rapporte une remarque à son adresse qu'elle a entendue lors d'un mariage algérien: "aucune vraie femme ne préférait la pollution d'une grande ville aux bras d'un homme» ( $P P$ 52). L'auteure de ces paroles critique ainsi le choix de l'exil et met en doute la féminité de l'héroïne qui a opté pour la vie à Paris au lieu de rester en Algérie et se marier conformément aux coutumes du pays.

La narratrice remarque également que, malgré un changement générationnel concernant la situation de la femme en Algérie et son acquisition de plusieurs droits, l'impératif de se marier n'a point changé. Elle commente ainsi les paroles de sa grand-mère: «Voter, conduire, travailler, voyager, déposer plainte... les femmes ont des droits maintenant, rétorquait [la grand-mère] et elle s'en réjouissait. Nous, c'était le mariage et c'est tout! Nous, c'est tout le reste et le mariage en plus. On n'a pas le droit de rester célibataire...» (PP 57). Cela revient à dire que malgré la Constitution qui octroie aux femmes de nombreux droits et malgré un choix beaucoup plus grand des activités qu'elles peuvent à présent exercer, le mariage reste leur obligation primordiale. La protagoniste remarque également que la lutte pour le changement de ce statu quo serait vaine: "L'Algérie et ses femmes. Les rêves de mariage. Les féministes, les carriéristes, les belles, les riches, presque toutes ont abandonné la cause. Elles veulent un homme, une jouissance, un statut. » (PP 54). L'héroïne énumère les catégories des femmes à qui le statut social devrait permettre de vivre aisément sans homme. Il semble pourtant que rien n'égale à un mari, ni carrière professionnelle, ni beauté, ni argent...

Nous voyons que, chez Adimi, à la différence du roman marouanien, l'accent est mis plus sur la nécessité de trouver un partenaire que de devenir mère. La narratrice se rend compte que, pour son entourage, le mariage constitue non seulement la voie unique pour la femme, mais encore un remède à tous les maux: «Mon médecin m'a fait remarquer que si j'avais un mari, celui-ci prendrait soin de moi» (PP 21), comme si une femme n'était pas capable de bien s'occuper d'elle-même. La remarque du médecin montre qu'en quittant l'Algérie la narratrice n'a pas réussi à échapper à la pression sociale et qu'elle la subit aussi en France. Elle dénonce entre autres le rôle des médias qui contribuent à la perpétuation de l'image négatif du célibat et qui éveillent le sentiment de culpabilité et de peur chez les célibataires: «Les magazines qui titrent qu'à partir de trente ans, une femme n'a plus qu'une chance sur cinq de rencontrer quelqu'un. [...] La revue de presse matinale qui égrène, à mon oreille, les suicides de femmes célibataires » (PP 103-104). Elle a l'impression que la Terre ne tourne qu'autour de la question du mariage : non seulement l'hérö̈ne doit supporter des remarques de ses parents proches et éloignés, regarder le bonheur de couples omniprésents dans l'espace public, mais encore elle est bombardée par des informations sur 
la mort de ses «semblables». L'auteure montre ainsi qu'il est impossible de fuir à la pression sociale qui peut avoir de conséquences néfastes pour la célibataire, mais aussi pour la société.

Premièrement, la peur de devenir une « vieille fille » ne permet pas à l'héroïne adimienne de fonctionner normalement. L'annonce des fiançailles de sa sœur, et qui plus est, une sœur cadette, qui ne devrait donc pas se marier en premier ( «Ça devrait être interdit, les petites sœurs qui se marient avant leur sœur aînée. Ça devrait être comme deux cousins qui s'unissent: quelque chose à la frontière de l'illégalité, quelque chose de malsain qui met mal à l'aise les honnêtes gens» ( $P P$ 25), remarque-t-elle), réveille des angoissent bien cachées ou tues. La protagoniste supporte très mal cette pression, elle est souvent saisie de panique causée par son célibat, mais aussi par sa solitude, intensifiée par l'éloignement de ses proches: «Je suis sortie, paniquée. Si. J'avais. Un. Mari» (PP 21); «Je tais mes frayeurs. [...] mon estomac est au bord du naufrage» (PP 49); «C'est Kaboul dans mon corps. Le Kaboul du début des années deux mille» (PP 128); « Je parle toute seule. Ma solitude est en train de grignoter mon corps. Mes angoissent prennent le contrôle de mon existence» ( $P P$ 137), avoue-t-elle.

Deuxièmement, la pression sociale force les femmes à participer dans une course pour trouver un mari. Souvent il ne s'agit pas de trouver un homme idéal ou un grand amour, mais de se faire épouser par le premier venu, comme le prouve la constatation de Hadda concernant son choix du futur partenaire: « Nassib n'était pas ce qu'on pouvait appeler un bel homme [...]. Mais là n'est pas l'essentiel! glapiraient mes voisines. Elles auraient raison, cent fois raison: un homme est un homme » (FC 44). La protagoniste qui voit les défauts tant de son physique que de son caractère, qui répugne même au contact physique avec lui, essaie de se convaincre d'avoir fait un bon choix. Malgré ses doutes, elle est persuadée que n'importe quel homme vaut mieux que le statut de «vieille fille». Cette conviction, largement répandue dans son entourage, contribue à cette poursuite des hommes.

Encore un autre personnage dans La fille de la Casbah, Mimouna, dresse le même diagnostic en disant: «Ils [les hommes] sont tous pareils. Et ils disent la même chose de nous. [...] Que nous sommes toutes à courir après le mariage. Ils n'ont pas vraiment tort» ( $F C$ 33). Certes, Hadda rétorque à sa cousine qu'« il n'y a pas que le mariage dans la vie» (FC 36), mais par son attitude et ses actes elle confirme l'opinion répandue sur le besoin de la femme algérienne à se faire épouser. La même conviction apparaît dans le roman d'Adimi, cependant l'auteure tient à souligner encore une fois qu'il ne s'agit pas d'une particularité algérienne ou maghrébine et que les Françaises, avec toute leur émancipation, sont très semblables dans leur attitude envers le mariage: «Des deux côtés de la Méditerranée, les femmes agissent de façon identique quand elles nourrissent l'espoir de trouver l'homme de leur vie: avec désespoir, des calculs machiavéliques en tête et un brin de naïveté » (PP 55-56). La narratrice peut paraître sé- 
vère dans son jugement des jeunes filles quand elle les accuse tant d'utiliser des stratagèmes pour piéger un homme, tant d'être niaises et désespérées à la fois.

Par ailleurs, elle ne se montre pas plus indulgente envers les femmes mariées à qui elle reproche des imperfections physiques en s'étonnant qu'elles soient toutes mariées: celle-là a une «bouche de grenouille rouge vif», celle-ci est «plate comme un fil de fer», une autre «sent mauvais» (PP 50)... Malgré cela elles ont toutes une «bague à l'annulaire gauche» $(P P$ 50), tandis qu'« [elle]: annulaire vide» (PP 51). Ce doigt destiné par les lois sociales à informer du statut civil de l'individu devient le symbole de son échec, de son fiasco sur le marché matrimonial. «Certains jours, je trouve mon annulaire gauche plus gros que les autres doigts. Je l'imagine se glissant dans ma gorge et me coupant la respiration» ( $P P$ 58). Ainsi l'héroïne d'Adimi semble-t-elle ne se définir qu'en fonction de l'autre, de la femme mariée, pour laquelle elle éprouve à la fois de la jalousie et du dédain. Cette distanciation ironique fait ressortir son amertume, son sentiment d'injustice à cause de ce célibat involontaire. Ainsi, nous pouvons constater une autre conséquence de la pression sociale et des attentes qu'on ne peut pas satisfaire : le sentiment d'échec et l'amertume de la célibataire qui se tournent contre les personnes qui ont réussi, dans ce cas contre toutes les femmes mariées.

Adimi avertit encore d'un autre danger, beaucoup plus lourd de conséquences, de la pression sociale omniprésente. Elle montre à quel point celle-là peut s'avérer invivable pour les femmes qui la subissent en se servant de deux faits divers inventés entrelacés au récit: le premier à l'ouverture du roman «Une jeune femme de trente ans a été arrêtée pour le meurtre de sa voisine qui s'était moquée d'elle en déclarant qu'elle ne trouverait jamais d'homme assez fou pour l'épouser» $(P P 9)$; et le second, plus tard - l'histoire d'une jeune femme qui a assassiné sa meilleure amie parce que celle-ci lui avait reproché «de ne pas faire de véritables efforts pour trouver quelqu'un » ( $P P$ 113). Ces deux crimes anecdotiques montrent que les femmes célibataires sont constamment exposées aux remarques, sarcastiques ou même bienveillantes, concernant leur célibat. «J'aurais fait pareil» ( $P P$ 9), avoue la narratrice, compatissant les deux meurtrières. L'image de la «vieille fille» qui ressort de ces faits divers confirme la remarque de Françoise HérITIER que le célibat est considéré comme «une menace mystique pour la société» (1996: 242) et que les femmes célibataires sont jugées dangereuses non seulement pour elles-mêmes, mais également pour la collectivité (1996: 251).

Il est également à noter que la protagoniste ne croit pas faire partie de ce triste «club» de «vieilles filles». «Ma mère méprise les vieilles filles. Et moi aussi à vrai dire. Je les imagine comme des femmes au corps vide et au visage inexpressif. Elles sont inutiles. Elles ont raté leur tour [...]. N'importe quel mari dans son lit vaut mieux que pas de mari du tout.» (PP 58). Dans son discours, la «vieille fille» n'est pas une femme réelle, en chair et en os, mais un fantôme, 
un être imaginaire dénué d'émotions, un robot. Elle est aussi tenue responsable de sa situation déplorable. Ce qui choque peut-être le plus dans cette vision, c'est la constatation de l'inanité de cette partie de la population. Cela démontre l'intériorisation par la protagoniste de la représentation collective de la célibataire en tant qu'une personne sans valeur et sans intérêt pour la société, comme si son existence entière se résumait à être une femme de quelqu'un et non une femme tout court.

On retrouve également dans le texte adimien l'idée reçue la plus caricaturale sur la célibataire en tant que propriétaire d'un ou de plusieurs chats qui, selon certaines légendes urbaines, mourrait en solitude et servirait de nourriture à son animal: «Je pense aux statistiques qui sont contre moi dans cette grande ville française et aux femmes qui meurent seules, ou pire, avec un chat dévoreur de visage. On les découvre six jours plus tard » ( $P P$ 21). Prendre un animal, surtout un chat, apparaît comme un stade ultime du célibat, la frontière après laquelle les chances de trouver un mari s'approchent dangereusement du zéro, ce que la mère de la narratrice craint pour sa fille aînée: "'Ta petite sœur va se marier et toi, tu restes seule... Il ne te manque plus qu'un chat! Une vieille fille et son chat!' [...] À chaque fois, elle me parle de ce satané chat» (PP 164). Le félin devient le symbole de la solitude et de la folie, comme le laisse entendre l'appellation anglaise de cette figure féminine, à savoir crazy cat lady.

Néanmoins, la protagoniste n'adhère pas à ce rôle et se révolte finalement contre le mariage qui lui est imposé par son milieu. Comme le remarque de justesse Sabrina Fatmi, «[m]arginalisée de par son statut de 'vieille fille', l'héroïne tentera inlassablement de franchir les frontières psychologiques qui lui sont imposées pour ainsi récuser toute forme de subjugation, en faisant de sa marginalité un nouveau centre et en se positionnant à la jonction de deux mondes» (FATMI, 2019). La narratrice avoue elle-même être une «barre médiane» ( $P P$ 76), demeurer dans un (ou plusieurs) entre-deux : entre l'Algérie et la France, entre les traditions et la modernité, entre la famille qui lui manque et sa vie douillette parisienne, entre ce que veut d'elle sa mère/la société et ses propres rêves et aspirations. Les imminentes fiançailles de sa sœur l'amènent à affronter son propre regard, à s'interroger sur son identité et à décider ce qu'elle espère vraiment de sa vie. «Je désire [...] quelqu'un de magique et d'étrange qui me rappellerait la petite maison du bord de mer et les traînées de sel sur les épaules» (PP 145) elle se rend compte qu'elle ne pourrait pas se contenter d'un homme quelconque, le premier venu, seulement pour ne pas rester seule. Elle cherche un vrai amour malgré les attentes des autres qui pèsent sur elle, malgré ce qui l'attend en Algérie lors de la cérémonie familiale, elle fait son choix et trouve enfin la paix. Elle s'imagine l'avenir: "Un jour, je reviendrai à Alger et ce ne sera pas un drame» (PP 174).

Tandis que chez Adimi la rupture avec le célibat ne découle pas de la nécessité intérieure de l'héroïne qui se plaît dans sa «douillette vie parisienne» 
( $P P$ 76), mais se présente plutôt comme un moyen pour exaucer le vœu de sa mère, chez Marouane elle constitue la seule opportunité de réussir l'ascension sociale et d'échapper à la misère dans laquelle sombre la protagoniste et sa famille. La juxtaposition de ces deux histoires montre que, malgré la distance temporelle de trente ans et tous les changements survenus, les stéréotypes sont restés inchangés, ce qui confirme la thèse selon laquelle l'inaltérabilité constitue un des traits caractéristiques du stéréotype.

Marouane et Adimi dressent dans leurs romans des portraits de jeunes femmes célibataires, ainsi que de différents acteurs sociaux réagissant à leur statut. En se servant de moyens stylistiques différents - chez Marouane la poétique réaliste au début et onirique vers la fin, chez Adimi, réaliste, mais remplie de l'humour tranchant tournée tant vers la narratrice elle-même, tant vers la réalité qui l'entoure -, les deux écrivaines réussissent à formuler une critique sévère de la société algérienne contemporaine qui maintient le statu quo en cantonnant les femmes dans des représentations stéréotypées et en stigmatisant celles qui ne se conforment pas aux normes sociétales. Dans notre corpus, la lutte pour la libération de la femme de ce joug passe entre autres par la dénonciation du rôle assigné traditionnellement à la femme et par la ridiculisation et la contestation du stéréotype de la célibataire, la vieille fille, la baïrra.

\section{Bibliographie}

Adimi, Kaouther 2016: Des pierres dans ma poche. Paris, Seuil.

Amossy, Ruth 1989: «La notion de stéréotype dans la réflexion contemporaine». Littérature, $\mathrm{n}^{\circ}$ 73: Mutations d'images, p. 29-46< <ttps://www.persee.fr/doc/litt_0047-4800_1989 num_73_1_1473> Date de consultation: le 25 février 2019.

Amossy, Ruth; Herschberg Pierrot, Anne 1997: Stéréotypes et clichés: langue, discours, société. Paris, Nathan.

Charpentier, Isabelle 2012: «Rituel de protection de la virginité féminine et nuit de sang dans la littérature (franco-)algérienne». In: Penser le corps au Maghreb. Sous la dir. de Monia LACHheB. Paris, Tunis, Karthala et IRMC, p. 201-216.

Charpentier, Isabelle 2015: «De la difficulté (sexuelle) d'être une femme célibataire au Maghreb : une étude de témoignages et d'œuvres d'écrivaines algériennes et marocaines ». Modern \& Contemporary France, Vol. 23, Issue 4, p. 435-455 <http://www.tandfonline.com/ doi/full/10.1080/09639489.2015.1037726>. Date de consultation: le 15 novembre 2015.

Chebel, Malek 2003 : L'esprit de sérail. Mythes et pratiques sexuels au Maghreb. Paris, Payot \& Rivages. 
DuFAYs, Jean-Louis 1994: «Stéréotype et littérature: L'inéluctable va et vient». In: Le Stéréotype: crise et transformations [en ligne]. Caen: Presses universitaires de Caen, p. 77-89 $<$ http://books.openedition.org/puc/9702>. Date de consultation: le 25 février 2019.

El NosSERY, Névine 2012: Témoignages fictionnels au féminin. Une réécriture des blancs de la guerre civile algérienne. Amsterdam, New York, Rodopi.

Fatmi, Sabrina 2019: «La traversée des frontières dans l'œuvre de Kaouther Adimi: à la recherche du nouveau rôle féminin?» (communication orale lors du colloque Women of the Mediterranean, Sant'Anna Institute, Sorrento 21-22 juin 2019).

GoulEt, Alain 1994: «L'écriture du stéréotype dans la littérature contemporaine». In: Le Stéréotype: crise et transformations [en ligne]. Caen: Presses universitaires de Caen, p. 181-201 <http://books.openedition.org/puc/9711>. Date de consultation: le 25 février 2019.

HÉRITIER, Françoise 1996: Masculin/féminin. La pensée de la différence. Paris : Odile Jacob.

MalinowsKa, Magdalena 2019: «Le rôle de la scolarisation des filles dans l'émancipation des femmes algériennes dès l'indépendance jusqu'au XXI ${ }^{e}$ siècle ». In : Femmes et le savoir : histoire, défis, perspectives. Sous la réd. de Joanna Godlewicz-Adamiec, Dariusz Krawczyk, Małgorzata Łuczyńska-HoŁdys, Paweł Piszczatowski, Małgorzata SokoŁowicz. Paris, Classiques Garnier.

Maroune, Leïla 1996: La Fille de la Casbah. Paris, Julliard.

Perrig-Chiello, Pasqualina 2001: «Images sexuées de la vieillesse : entre stéréotypes sociaux et auto-définition». Retraite et société, 3 (n $\left.\mathrm{n}^{\circ} 34\right)$, p. 69-87. <https://www.cairn.info/revueretraite-et-societe1-2001-3-page-69.htm>. Date de consultation : le 27 février 2019.

Rude-Antoine, Edwige 1990: Le mariage maghrébin en France. Paris, Éditions Karthala.

Willems, Emilio 1970: Dictionnaire de sociologie. Adaptation française par A. Cuvillier. Librairie Marcel Rivière et Cie.

\section{Note bio-bibliographique}

Magdalena Malinowska - maîtresse de conférences à l'Institut des Langues Romanes et de Traduction à l'Université de Silésie à Katowice. Elle travaille sur la littérature et la culture maghrébines et arabes, sur le féminisme contemporain, les études sur le genre et la sexualité. À présent, elle consacre sa recherche aux questions liées à la violence faite aux femmes en temps de guerre (notamment lors de la décennie noire algérienne).

magdalena.malinowska.us@gmail.com 